\title{
La evaluación en las materias Gráfico Visual de la Universidad de Santiago de Compostela
}

\section{Evaluation of Visual Graphic courses at the University of Santiago de Compostela}

\author{
Vicente López-Pena *, Andrea M. López-Chao **, Vicente A. López-Chao** \\ *Universidad de Cádiz, **Universidade da Coruña
}

\begin{abstract}
Resumen
La evaluación es una actividad compleja que forma parte del proceso educativo y siempre ha constituido una preocupación central del docente. Para averiguar cómo se realiza en las titulaciones técnicas de la Universidad de Santiago que contienen entre sus materias alguna relacionada con la Enseñanza Gráfico Visual se han analizado sus guías docentes. Los resultados indican que de las 12 asignaturas de Expresión Gráfico Visual presentes en la USC, sólo en $2(16,6 \%$ de la muestra) se detalla el modo de realizar la evaluación, siendo en ambos casos el examen el responsable del 60 o $65 \%$ de la nota final.
\end{abstract}

Palabras clave: Evaluación, examen, Expresión Gráfico Visual, Universidad de Santiago.

\begin{abstract}
Evaluation is a complex activity that is part of the educational process and has always been a main concern for teachers. This research was conducted through an analysis of the educational guides of all technical degrees from the University of Santiago taking into account every course dealing with Visual Design in order to find out what are the evaluative strategies used. The results, which indicate that out of the 12 subjects of Visual Graphic Expression present at USC, only 2 (16.6\% of the sample) detail how to conduct the assessment, and in both cases the exam accounts for 60 or $65 \%$ of the final grade.
\end{abstract}

Keywords: Evaluation, exam, visual-graphic expression, USC

\section{EI EEES y la renovación de las metodologías de enseñanza}

Las sucesivas declaraciones firmadas y aceptadas por los Estados miembros desde la declaración de Bolonia del año 1999 fueron definiendo las modificaciones que se debían realizar con vistas a convertirse en realidad el año 2010, exigiendo a las Universidades realizar la mayor trasformación de las últimas décadas. Entre las transformaciones a realizar, se encuentran los métodos de enseñanza, que deben, a partir de este momento, favorecer la formación integral de los estudiantes basada en la importancia de su trabajo (Angulo, Corpas, García \& González, 2006).

En un primer momento no se planteó una concepción sobre los perfiles del diseño final, pero sí se hizo evidente la necesidad ineludible e inaplazable de cambio en la orientación metodológica (Sánchez, 2010). Pero la declaración de Glasgow del año 2005, en el apartado denominado "Reenfoque del proceso de Bolonia a medio camino de 2010", plantea la reorientación de los currículos por medio del diálogo con los empresarios, asumiendo el reto de una educación académica, profesional y permanente, y además se señala que las Universidades deben comprometerse a duplicar sus esfuerzos para "introducir métodos de enseñanza innovadores" (EUA, 2005:15). En las universidades españolas la puesta en marcha del EEES ha significado nuevos planes de estudios y sobre todo un cambio en las metodologías docentes y en cómo se valora a los estudiantes.

Es decir, la incorporación de las universidades españolas al EEES significó una serie de adaptaciones no solo legislativas, sino que primordialmente modificó el rol tanto del docente como del discente, pues según indica Martín Delgado (2011), el objetivo de la enseñanza universitaria desde la entrada en el EEES fue la profesionalidad del aprendizaje, con la finalidad de que los estudiantes se integren al mercado laboral, para lo cual se contemplan las competencias genéricas básicas y transversales relacionadas con la formación integral de la persona y competencias específicas en función de la materia. Constituyó, pues, un cambio esencial en el modo de concebir la enseñanza universitaria, al menos en los países como España, que a juicio de Clavo \& Mingorance (2009: 320) utilizaban un sistema pedagógico de tradición napoleónica, progresando hacia cambios metodológicos y nuevos sistemas pedagógicos para cuando estos sean obligatorios a partir del año 2010. Este nuevo escenario no ha estado exento de críticas. Determinadas voces se alzaron para denunciar que se trata de "forzar a las universidades y centros de investigación públicos para ponerse por completo al servicio de las necesidades de los mercados y empresas capitalistas" (Torres Santomé, 2011:185).

Según Pérez et al. (2008) estas transformaciones tienen un doble objetivo, dado que junto con formar un sistema de educación superior que mejore el empleo y la movilidad de los ciudadanos aumentará también la competitividad internacional de la educación superior a nivel europeo, facilitando el intercambio de titulados, estudiantes y docentes gracias a la adaptación de los contenidos de los estudios universitarios a las demandas sociales. Sin embargo Zabalza (2003:8) considera que el nuevo EEES prepararía al estudiante para el mercado laboral indicando que este "ha abierto nuevos cauces para definir la orientación de los estudios de forma que estos se adecuen a las condiciones y demandas que plantea la 
sociedad del conocimiento y su consecuencia inmediata de formación a lo largo de la vida".

En definitiva, el ingreso en el nuevo EEES implicó realizar "una reflexión, no solo individual, sino también conjunta en lo que se refiere a las tres tareas fundamentales e interrelacionadas de la intervención educativa: la planificación, la ejecución de la enseñanza y la evaluación" (García \& Morillas, 2011:114).

La introducción de las competencias en el sistema universitario impulsó profundos cambios en relación a las metodologías aplicadas, técnicas de evaluación y la función ejercida por los docentes, que ha de servir de guía en la adquisición de las competencias requeridas (Pérez et al. 2008). Formar en competencias implica un cambio en la forma de pensar, sentir y actuar del estudiante, obligando al docente a cambiar el enfoque educativo que debe orientarse a quién aprende con mayor participación y respondiendo a las demandas crecientes de la sociedad (Mingorance \& Calvo, 2013). La formación por competencias se basa en el reencuentro del cognitivismo y el constructivismo. El primero se ocupa que el estudiante adquiera y aplique conocimientos y habilidades favoreciendo la construcción gradual de los conocimientos, tomando en cuenta elementos afectivos, cognitivos y meta cognitivos. El constructivismo en tanto hace hincapié en el estudiante, fomentando su autonomía, presentándole tareas significativas en las cuales pueda manipular materias e interactuar con los demás. El docente guía para que el estudiante construya sus conocimientos, su saber ser y su saber hacer (Fernández March, 2011).

La enseñanza tradicional centrada en la trasmisión de conocimientos al estudiante es sustituida por este nuevo modelo basado en competencias, ya que "lo importante no es tanto la posesión de una serie de conocimientos como el uso que se haga de ellos" (García \& Morillas, 2011:117) impulsando al docente universitario a replantear su actuación. Ha supuesto un desafío importante para los docentes durante el proceso de convergencia transitar hacia una enseñanza basada en el desarrollo de competencias, un modelo en el que prevalece el proceso de aprendizaje en el estudiante, diferenciándose así de la escasa participación que este tenía en el modelo tradicional. El docente debe garantizar en sus estudiantes el desarrollo de estos tres aspectos que componen el concepto de competencias de manera íntegra: Conocimientos, habilidades y actitudes (saber, saber hacer y saber ser y estar).

En definitiva, el EEES representó una magnífica oportunidad para replantear la temática de las metodologías educativas apuntando a la mejora del aprendizaje de los estudiantes e incrementando su nivel de satisfacción y motivación al igual que en los docentes. En este proceso de modernización no tiene cabida la metodología tradicional, donde el peso de la clase recaía sobre el docente, más preocupado de lo que debe enseñar, dando paso a una metodología centrada en el estudiante y como aprende. Además deja de depender exclusivamente de los conocimientos teóricos, que si bien siguen siendo esenciales, no son lo único (...) requerirá que, las lecciones magistrales, de las que tanto ha abusado el sistema educativo español, sean complementadas con seminarios y talleres que fomenten el desarrollo de la responsabilidad, el trabajo en equipo, el liderazgo, y la formación humana del estudiante (Mingorance, 2008: 99-100).

Evidentemente, este proceso requirió un profundo reciclaje del profesorado. Pero a pesar de que ha existido disposición e interés de los docentes por participar en cursos de formación, ello no aseguró necesariamente que estos estén preparados y dispuestos a implementar en sus estudiantes estas nuevas metodologías y además que existan las condiciones para ello (Andreu \& Labrador 2011).

\section{La evaluación en función de las nuevas metodologías y la adquisición de competencias}

En este contexto es evidente que la transformación no puede afectar sólo a las metodologías de enseñanza. Así pues, en su informe "Criterios y Directrices para la Garantía de Calidad" en el EEES (2005) la European Association for Quality Assurance in Higher Education (ENQA) indica que uno de los elementos más importantes de la educación superior es la evaluación, debido a que sus resultados tienen efectos profundos sobre la evolución curricular del estudiante, permitiendo además a las instituciones conocer la efectividad de la enseñanza entregada. Se recalca, por tanto, la importancia de que se efectúe de un modo profesional, disponiendo de un extenso conocimiento de los procesos de pruebas y exámenes, docentes que comprendan el trascendental rol de la evaluación en la progresión de sus estudiantes hacia la adquisición no solo de conocimientos, sino también de habilidades. La CRUE describe en el documento "Efecto del EEES en el ámbito de los estudiantes" (2005) que uno de los efectos de la convergencia europea en la actividad académica es obligar a un replanteamiento y diversificación de las técnicas y sistemas de evaluación. Ya no se evaluará exclusivamente el nivel de conocimientos, sino también la adquisición de otras habilidades, destrezas y aptitudes por parte de los estudiantes. En este contexto, el examen clásico de conocimientos perderá peso en la evaluación global y, en general, la superación de dicho examen no será condición suficiente para superar la asignatura. (CRUE, 2005:4).

Consideramos que la evaluación es una actividad compleja que forma parte del proceso educativo y siempre ha constituido una preocupación central del docente. Aunque a veces es percibida como el componente más incómodo del proceso de enseñanzaaprendizaje, resulta esencial contar con un sistema de evaluación que legitime y promueva la calidad. (Álvarez, 2008; Biggs, 2005). Para Geli (en Rodríguez, Ibarra, Gallego, Gómez \& Quesada, 2012) evaluar es recoger información desde el inicio hasta el final del proceso de enseñanza-aprendizaje para analizarla e interpretarla. En esta línea Bain (2006) considera que la evaluación no se entiende como una actividad final del proceso de enseñanza-aprendizaje, sino como un instrumento para favorecer el aprendizaje, que debe ser continua, y sirva para que el docente ayude al estudiante. La evaluación por tanto es "un proceso sistemático, intencional y 
continuo de recogida de información, supone análisis, interpretación y valoración de la misma, en base a criterios, que conduzcan a una toma de decisiones en relación al objeto evaluado" (García \& Morillas, 2011:119). Esta no constituye una labor que se realiza al margen del proceso de enseñanza-aprendizaje, siendo una actividad integrada la acción docente.

A nuestro juicio la evaluación es uno de los elementos clave del proceso formativo en cualquier nivel educativo, sus finalidades son amplias y no se remiten solamente a calificar, clasificar o aprobar estudiantes o que este sea capaz simplemente de reproducir la información asimilada (Zabalza, 2003). Dada su importancia, las formas de evaluación y sus estrategias asociadas han de ser variadas, además de constituir una fuente de información lo suficientemente amplia como para calificar a los estudiantes, puesto que no sería responsable pretender evaluar las tres dimensiones del aprendizaje empleando solo las formas correspondientes a una de estas dimensiones. Es decir, la convergencia hacia el EEES implicó el replanteamiento de buena parte del modo de actuar del docente universitario y en especial de la evaluación, dado que esta se considera ahora como una parte más del proceso de aprendizaje (Álvarez, Fernández de Haro \& Perales, 2007). Marcó diferencias evidentes con respecto a la "trasnochada concepción restrictiva de la evaluación, que le convierte en un mero apéndice de proceso de enseñanza-aprendizaje" (López, 2007:17), quedando integrada en diversos momentos de este. El EEES permitió la obtención de los títulos basados en una evaluación continua más que en los tradicionales exámenes finales, que elevan de forma artificial el riesgo que los estudiantes suspendan (Reichert \& Tauch, 2003 y 2005).

Por todo lo expuesto, consideramos que el sistema de evaluación puntual tradicional ha de ser sustituido, sin embargo todavía queda mucho por hacer, pues un estudio realizado por Rodríguez et. al. (2012) a un grupo de estudiantes concluye que aún predomina la evaluación tradicional y el escaso uso de estrategias participativas en la evaluación.

\section{Objetivos}

El objetivo principal de esta investigación es averiguar qué métodos de evaluación se utilizan en las titulaciones de la USC que contienen entre sus materias alguna relacionada con la Enseñanza Gráfico Visual. Para llevar a cabo esta investigación hemos realizado un análisis de las guías docentes de todas las materias relacionadas con el constructo objeto de estudio, utilizadas por los profesores para el desarrollo de su labor docente, y descargadas de las distintas WEBs de las Facultades de la Universidad de Santiago.

Hemos comenzado realizando un estudio de los centros existentes en los que se imparten Grados en Ingeniería o Arquitectura en los diferentes Campus Universitarios de dicha Universidad.

La muestra la constituyen 7 titulaciones de Grado en Ingeniería que contemplan materias de Expresión Gráfico Visual.

\section{Resultados}

Tras el análisis de la guías docentes de las materias de Expresión Gráfico Visual de la Universidad de Santiago se desprende que los sistemas de evaluación empleados por los docentes son: Clases Magistrales (C.M.), Sesiones Prácticas de Laboratorio (Lab.), Seminarios (Sem.), Prácticas (Prác.), Tutorías Grupales (T.G.) Tutorías Individuales (T.I.), Trabajos (Trab.), Campus Virtual (C.V.), Asistencia a Clase (A.) y Evaluación (E.), que no corresponden con las metodologías empleadas (Tabla 1).

Tabla1.

Comparativa entre metodologías y estrategias de evaluación en la USC

\begin{tabular}{|c|c|c|c|c|c|c|c|c|c|c|c|}
\hline \multirow[b]{2}{*}{ Títulación } & \multirow[b]{2}{*}{ Asignatura } & \multicolumn{9}{|c|}{ Sistema de evaluación en USC } & \multirow[b]{2}{*}{$\bar{E}$} \\
\hline & & C.M & $\overline{\mathrm{Lab}}$ & $\overline{\text { Sem }}$ & $\overline{\text { Prác }}$ & TG & T I & Trab & C. V. & $\bar{A}$ & \\
\hline 1, $-G_{\text {, en Ing, Quimica }}$ & 1.-Expresión Gráfica & 0 & $\overline{0}$ & 0 & & 0 & 0 & $40 \%$ & 0 & & $60 \%$ \\
\hline 2.- G. en Proc, Qum Indust & 2.-E. Gratica y DAO & 0 & $\pi$ & $\pi$ & & $\pi$ & 0 & $35 \%$ & 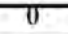 & & $65 \%$ \\
\hline \multirow[t]{2}{*}{ 3.- G. en Ing. Agr y del M. Rural } & \multirow{2}{*}{$\begin{array}{l}\text { 3.1.-E. G en la Ing. } \\
\text { 3,2,-CAD. E.G.A }\end{array}$} & ¿? & & ¿? & ¿? & & & & & ¿? & \\
\hline & & 0 & 0 & & ¿' & & & & & & d? \\
\hline \multirow[t]{2}{*}{ 4,- G. en Ing. de las Ind Agr } & \multirow{2}{*}{$\begin{array}{l}\text { 4.1. E. Genla Ing. } \\
\text { 4.2.-C.AD. E.G.A. }\end{array}$} & ¿? & & ¿? & हर & & & & & $+\frac{1}{4}$ & \\
\hline & & 0 & 0 & & ¿? & & & & & & ¿? \\
\hline \multirow[t]{2}{*}{ 5.- G. en Ing, $1 . y$ deाM. N. } & \multirow{2}{*}{$\begin{array}{l}\text { 5.1.-E.G. en la Ing. } \\
\text { 5.2.-CAD. E.G.A }\end{array}$} & ¿? & & ¿? & $e^{?}$ & & & & & है? & \\
\hline & & 0 & 0 & & ¿? & & & & & & ¿? \\
\hline \multirow[t]{2}{*}{ 6.- G. en Ingeniería Civil } & \multirow{2}{*}{$\begin{array}{l}\text { 6.1.-E. G. en Ingeniería } \\
\text { 6.2.-D. T. A. O. }\end{array}$} & $\overline{0}$ & & 0 & ¿? & 0 & 0 & $\overline{0}$ & 0 & & $\dot{d}^{?}$ \\
\hline & & 0 & & & ¿द & 0 & & & & & $\sum^{2}$ \\
\hline \multirow[t]{2}{*}{ 7, G. en Ing. Geom y Topo. } & \multirow{2}{*}{$\begin{array}{l}\text { 7.1.-Sistemas de Repre. } \\
\text { 7.2.-D. Téc. Asis por O. }\end{array}$} & 0 & 0 & & & 0 & 0 & $e^{?}$ & & & $\AA^{?}$ \\
\hline & & & $c^{?}$ & & & 0 & 0 & & & & द? \\
\hline
\end{tabular}

En la Tabla 1 se indican las metodologías que el profesorado declara utilizar y la cuantificación de los sistemas de evaluación que emplean, que deberían corresponderse con las metodologías, aunque no es así, como se puede apreciar en la Figura 1.

Como se puede observar, en las dos primeras materias, en aquellas casillas en la que en el tipo de metodología utilizado aparecía señalado, y sin embargo no se ve reflejado en la evaluación, lo hemos distinguido en la tabla con un 0 . En las diez restantes se especifican cuáles son las estrategias de evaluación, aunque no aparece ponderación alguna. Para diferenciar este supuesto de aquellos en los que no se contempla, hemos recurrido a señalarlos con dos interrogaciones. Es decir, en la asignatura CAD. Expresión Gráfica Aplicada, del Grado en Ingeniería Agrícola y del Medio Rural, de las cuatro 
metodologías señaladas (Clase Magistral, Laboratorio, Prácticas y Examen) sólo las dos últimas se ven reflejadas en la evaluación, aunque no se indica cuál es la ponderación de cada una de ellas.

Figura 1. Comparativa entre metodologías y estrategias de evaluación en la USC

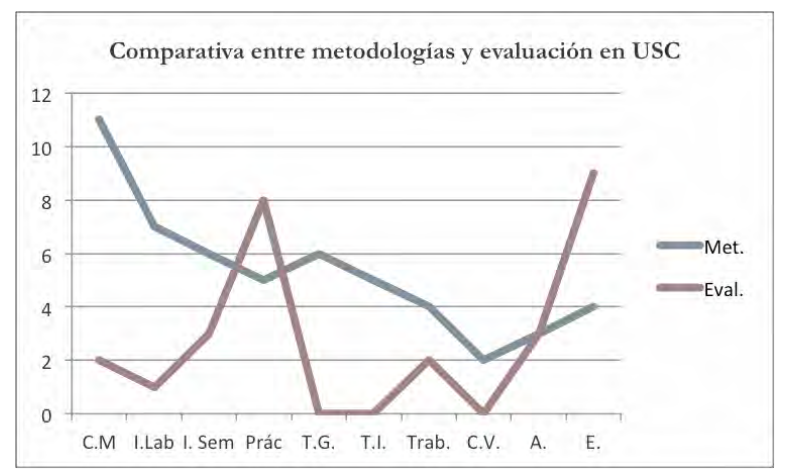

Teniendo esto en cuenta, de las 12 asignaturas de Expresión Gráfico Visual de la USC, sólo en 2 (16,6\% de la muestra) se detalla el modo de realizar la evaluación, siendo en ambos casos e examen el responsable del 60 o $65 \%$ de la nota final. Es resto de la calificación se realiza a través de los trabajos. Cabe destacar que a pesar de no estar detallada su ponderación, en el $75 \%$ de los casos se utiliza el examen como parte de la evaluación, y el $66,66 \%$ las prácticas.

\section{Conclusiones}

En primer lugar es muy significativo que sólo el 16,6\% del profesorado especifica en su guía docente el modo concreto en que será evaluado, lo que supone una indefensión del alumnado, que en todo momento debe conocer el modo en que va a ser evaluado y el peso específico que tendrá cada una de las pruebas.

Por otro lado, cabe destacar que a pesar de que se emplean variadas metodologías de enseñanza, no todas ellas están contempladas dentro de la evaluación, pues a pesar de que el plan Bolonia está totalmente instaurado, y la evaluación ha de incluir "un conjunto de actividades variadas y son numerosos los autores que defienden la necesidad de realizar estrategias de evaluación variadas que permitan conocer, desde distintas ópticas, el grado de aprendizaje que se está alcanzando y que le preparen para su salida al mercado de trabajo" Mingorance (2008:95), esto no se cumple en la Universidad de Santiago, donde la mayor parte del profesorado emplea dos estrategias para evaluar a sus discípulos, a pesar de ser variadas las estrategias empleadas.

Es también importante señalar que el tradicional examen sigue siendo el responsable de la mayor parte de las evaluaciones.

En definitiva, es inexcusable que si se propugna un cambio de metodología en la enseñanza aprendizaje, se produzca también un cambio en la evaluación. Es precisamente esta variable la que necesita que se le preste más atención. Para ello, será necesario utilizar la evaluación formativa que potencie lo que se sabe, la creatividad y el esfuerzo, más que la simple visión de juicio, positivo o negativo, de lo realizado por el alumno, pues a pesar de tantas reformas, todavía se está utilizando un tipo de evaluación que si bien puede informar de los conocimientos, no se ajusta a los nuevos requerimientos más amplios del EEES.

\section{Referencias}

Álvarez, I. (2008). Evaluación del aprendizaje en la universidad: Una mirada retrospectiva y prospectiva desde la divulgación científica. Electronic Journal of Research in Educational Psychology, 6(14), 235-271.

Álvarez, M., Fernández De Haro, E. y Perales, J. (2007). La enseñanza, el aprendizaje y el programa como ámbitos del proceso evaluador, en López López, M.C. Evaluación de los procesos de enseñanza-aprendizaje en la universidad y su adaptación al espacio europeo de educación superior. (pp. 47-69).Granada: Universidad de Granada.

Angulo, J., Corpas, C., García J. D. \& González I. (2006). Las competencias de la titulación de psicopedagogía a nivel andaluz: investigando la opinión del profesorado, del alumnado universitario y de los profesionales de la orientación. Revista de Investigación Educativa, 24(2), 575-593.

Andreu, M. \& Labrador, M. (2011). Formación del profesorado en metodologías y evaluación. Análisis cualitativo. Revista de Investigación en Educación, 9(2), 236-245.

Bain, K. (2005). Lo que hacen los mejores profesores universitarios. Barcelona: Universitat de Valencia.

Biggs, J. (2006). Calidad del aprendizaje universitario. Madrid: Narcea.

Calvo, A. \& Mingorance, A. (2009). La estrategia de las universidades frente al Espacio Europeo de Educación Superior. Revista Complutense de Educación, 20(2), 319-342.

Conferencia de Rectores de las Universidades Españolas. (2005). Efecto del Espacio Europeo de Educación Superior en el ámbito de los estudiantes.

Fernández March, A. (2011). La evaluación orientada al aprendizaje en un modelo de formación por competencias en la educación universitaria. En Buján Vidales, K. La evaluación de competencias en la educación superior: Las rúbricas como instrumento de evaluación, 37-56. Sevilla: Eduforma.

García, M. \& Morillas, L. (2011). La planificación de evaluación de competencias en Educación Superior. Revista Electrónica Interuniversitaria de Formación de Profesorado, 14(1), 113-124.

López, M.C. (2007). Evaluar en la universidad y en el marco del Espacio Europeo de Educación Superior. En López, M.C. Evaluación de los procesos de enseñanzaaprendizaje en la universidad y su adaptación al Espacio Europeo de Educación Superior, 11-18. Granada: Universidad de Granada.

Martín Delgado, I. (2011). El sistema de evaluación en el ECTS. Textos de docencia OBSEI, 1, 25-44.

Mingorance Arnáiz, A. C. (2008). Análisis comparado entre los resultados de una evaluación continua y otra puntual. El caso de la asignatura de macroeconomía. Revista de Investigación Educativa, 26(1), 95-120.

Mingorance, A. C. \& Calvo, A. (2013). Los resultados de los estudiantes en un proceso de evaluación con 
metodologías distintas. Revista de investigación Educativa, 31(1), 275-293.

Pérez Pueyo, A., Tabernero Sánchez, B., López Pastor, V. M., Ureña Ortín, N., Ruiz Lara, E., Caplloch Bujosa, M., González Fernández, N. \& Castejón Oliva, F. J. (2008). Evaluación formativa y compartida en la docencia universitaria y el Espacio Europeo de Educación Superior: cuestiones clave para su puesta en práctica. Revista de Educación, 347, 435-451.

Reichert, S. \& Tauch, Ch. (2003). Tendencias III: Progreso hacia el Espacio Europeo de Educación Superior (AEU).

Reichert, S. \& Tauch, Ch. (2005). Tendencias IV: Universidades Europeas, puesta en práctica en Bolonia (AEU).
Rodríguez, G., Ibarra, M., Gallego, B., Gómez, M. y Quesada, V. (2012). La voz del estudiante en la evaluación del aprendizaje: Un camino por recorrer en la universidad. Revista Electrónica de Investigación y Evaluación Educativa, 18(2), 1-20.

Sánchez González, M. (2010). Técnicas docentes y sistemas de evaluación en educación superior. Madrid, España: Narcea.

Torres, J. (2011). La justicia curricular. El caballo de la Troya de la cultura escolar. Madrid, España: Morata.

Zabalza, M. (2003). Competencias docentes del profesorado universitario. Madrid: Narcea. 